\section{INTRATUMORAL DELIVERY OF HIGH POTENCY STING AGONISTS MODULATES THE IMMUNOSUPPRESSIVE MYELOID COMPARTMENT AND INDUCES CURATIVE RESPONSES IN CHECKPOINT-REFRACTORY GLIOBLASTOMA MODELS}

${ }^{1}$ Spencer Lea*, ${ }^{2}$ Chao-Hsien Chen, ${ }^{2}$ Genevieve Hartley, ${ }^{1}$ Rodney Cheng-En Hsieh, ${ }^{2}$ Michael Curran. ${ }^{1}$ The University of Texas MD Anderson Cancer Center UTHealth Graduate School of Biomedical Sciences, Houston, TX, United States; ${ }^{2}$ The University of Texas MD Anderson Cancer Center, Houston, TX, United States

Background Glioblastoma is an aggressive primary brain malignancy that is characterized by a highly suppressive tumor microenvironment, including myeloid-derived suppressor cells, tumor-associated macrophages, and brain-resident microglia, but lacking significant $\mathrm{T}$ cell infiltration. ${ }^{1}{ }^{2}$ This phenotype is reflected in the recently developed $\mathrm{QKi}^{-/-} \mathrm{Pten}^{-/-} \mathrm{P} 53^{-/-}$(QPP) tumor model, ${ }^{3}$ which we show is resistant to PD1 or CTLA-4 blockade, but sensitive to agonists of the innate immune sensor Stimulator of Interferon Genes (STING). We have previously shown that agonists of the innate dsDNA-sensing cGASSTING pathway are capable of proinflammatory repolarization in in vitro models of suppressive myeloid cells, although their function in the context of the Glioblastoma myeloid compartment in vivo remains poorly understood. ${ }^{4}$

Methods We utilized the synthetic cyclic di-nucleotide STING agonists IACS-8803 (8803) and ML-RR-S2-CDA (MLRR) to assess survival and tumor immune infiltrate functional reprogramming in two orthotopic transplantable human and murine Glioblastoma tumor models, U87 and the recently developed QPP8 (Qki ${ }^{--}$Pten $\left.^{-/} \mathrm{P}^{-/-}\right)$. Using in vitro models of M2polarized microglia, we investigated the ability of natural (2'3'cGAMP) and synthetic (MLRR and 8803) STING agonists to reverse immunosuppressive microglial polarization.

Results We found that intratumoral delivery of STING agonists significantly prolonged survival in the murine QPP8 orthotopic Glioblastoma tumor model, in contrast to checkpoint blockade which had no benefit on survival. In huNOG-EXL mice engrafted with human hematopoietic stem cells implanted with orthotopic U87 Glioblastoma, intratumoral delivery of STING agonists significantly prolonged survival and reduced expression of CD163 and CD206 on human tumor-infiltrating myeloid populations. Preliminary data suggests that in vitro suppressively-polarized microglia reduce expression of M2 functional markers, and increase expression of iNOS, PD-L1, CD80, and CD86 in a STING agonist potency-dependent manner.

Conclusions We found that STING agonists can induce curative responses in checkpoint-refractory murine Glioblastoma models and mediate significant extension of survival in a humanized mouse U87 xenograft setting. This prolonged survival is associated with a decrease in immunosuppressive M2 functional markers in human tumor infiltrating myeloid populations. Additionally, M2-polarized microglia demonstrated a reduction in M2 functional markers and upregulation of proinflammatory M1 markers following treatment with STING agonists. Together these results indicate that delivery of STING agonists can induce proinflammatory repolarization of the Glioblastoma myeloid stroma, including both infiltrating myeloid populations and brain-resident microglia, to drive prolonged survival in refractory models of Glioblastoma.

\section{REFERENCES}

1. Gabrusiewicz K, Rodriguez B, Wei J, et al. Glioblastoma-infiltrated innate immune cells resemble M0 macrophage phenotype. JCI Insight 2016;1(2).
2. Quail DF, Joyce JA. The microenvironmental landscape of brain tumors. Cancer Cell 2017:31(3):326-41.

3. Shingu T, Ho AL, Yuan L, et al. Qki deficiency maintains stemness of glioma stem cells in suboptimal environment by downregulating endolysosomal degradation. Nat Genet 2017;49(1):75-86.

4. Ager C, Boda A, Rajapakshe K, et al. (2021) "High potency STING agonists engage unique myeloid pathways to reverse pancreatic cancer immune privilege. JITC (in press)

Ethics Approval All experiments were conducted according to protocols approved by the University of Texas MD Anderson Cancer Center Institutional Animal Care and Use Committee.

http://dx.doi.org/10.1136/jitc-2021-SITC2021.763 